\title{
Consulta de enfermería y adherencia terapéutica del paciente en hemodiálisis
}

\author{
María Dolores Ojeda Ramírez, Inmaculada Caro Rodríguez, David Ojeda Ramírez, Ana García Pérez, Sonia \\ García Hita, Sergio García Marcos
}

Unidad de Hemodiálisis de la APES. Hospital de Poniente. El Ejido. Almería. España

\section{Resumen}

Introducción: Al entrevistar a un paciente en la sala de hemodiálisis se producen muchas interferencias en la comunicación ya que simultáneamente atendemos a otros pacientes y se presentan complicaciones que tendremos que resolver de forma inmediata. Mediante la consulta de enfermería se pretende evitar esto, y darle el valor y tiempo que tienen las intervenciones enfermeras que son parte del tratamiento. Debemos asegurar que el paciente recibe instrucciones terapéuticas correctamente escritas, revisarlas y verificarlas con él para poder hacer un ajuste de niveles de comprensión, además de motivarlo y corresponsabilizarlo para lograr la adquisición de conductas implicadas en su autocuidado.

Objetivo: Evaluar el efecto de la consulta de enfermería al paciente renal en hemodiálisis sobre el cumplimiento terapéutico.

Material y Método: Estudio observacional longitudinal prospectivo de cohorte. Muestra de 42 pacientes en programa de hemodiálisis desde enero a junio 2014. Los pacientes seleccionados para la consulta son aquellos para los que la investigadora del estudio es enfermera referente y colaboradora (Grupo A). Se recogen datos de las diferentes variables revisando las historias clínicas y mediante la entrevista con el paciente y cuidador principal.

Resultados: Encontramos diferencias significativas al comparar las variables estudiadas entre el Grupo A y

$$
\begin{gathered}
\text { Correspondencia: } \\
\text { M }^{a} \text { Dolores 0jeda Ramírez } \\
\text { C/ Piamonte 4, } 5^{\circ} \mathrm{H} .04720 \text { Aguadulce-Almería } \\
\text { E-mail: lolaojedasoy@hotmail.com }
\end{gathered}
$$

Grupo B (resto pacientes) en cuanto al fósforo y cumplimiento farmacológico, pero no se encontraron en cuanto al potasio aunque la tendencia es a mejorar los niveles.

Conclusiones: La consulta de enfermería mejora la adherencia terapéutica en dieta y medicación, relacionándose con un mayor tiempo de dedicación al tratamiento y mayor contacto con el cuidador principal.

PALABRAS CLAVE: consulta de enfermería; adherencia terapéutica; paciente renal; hemodiálisis

\section{Nursing consultation and therapeutic adherence of the hemodialysis patient}

\begin{abstract}
Introduction: When interviewing a patient in the hemodialysis room, there is a lot of interference in communication since we simultaneously attend to other patients and present complications that we should solve immediately. Nursing consultation is intended to avoid this and give the value and time that nurses have interventions, which are part of the treatment. We must ensure that the patient receives correctly written therapeutic instructions, check and verify them with him in order to adjust comprehension levels, motivate and co-responsibility for achieving the acquisition of behaviors involved in self-care.
\end{abstract}

Objective: To evaluate the effect of the nursing consultation on the therapeutic compliance in the renal patient on hemodialysis. 
Material and Method: A prospective longitudinal observational cohort study. Sample of 42 patients in hemodialysis program from January to June 2014. Patients selected for the consultation are those that the researcher of the study is referring nurse and collaborator (Group A). Data are collected from the different variables by reviewing the medical records and by interviewing the patient and primary caregiver.

Results: Significant differences were found when comparing the variables studied between Group A and Group B (rest patients) in terms of phosphorus and pharmacological compliance, but were not found in terms of potassium although the tendency is to improve levels.

Conclusions: The nursing consultation improves the therapeutic adherence in diet and medication, being related to a greater time of dedication to the treatment and greater contact with the main caregiver.

KEYWORDS: nursing consultation; therapeutic adherence; renal patient; hemodialysis.

\section{Introducción}

Predominio e importancia del cumplimiento terapéutico en el paciente renal en programa de hemodiálisis (HD).

Los pacientes en HD soportan una situación de enfermedad generalmente durante largo periodo de tiempo, por lo que resulta más difícil el cumplimiento de las diferentes medidas terapéuticas ${ }^{1}$.

El hecho de que estos pacientes sean crónicos y generalmente polimedicados, genera numerosas dificultades para cumplir correctamente los tratamientos prescritos, por esta razón es fundamental fomentar la motivación y control de estos enfermos para que se cumpla mejor su régimen terapéutico ${ }^{2}$. Suelen ser pacientes con un mal cumplimiento terapéutico por una falta de información y/o comprensión sobre el tratamiento farmacológico, por la cronicidad y la pluripatología que favorece la desmotivación del paciente y también, por el hecho que se da en estos pacientes, de incumplir el tratamiento cuando se encuentran controlados y creen estar mejor ${ }^{3}$.

Las investigaciones de Cvengros, Chrisstensen y Lawton ${ }^{4}$ en 2004 ponen de manifiesto que la escasa adherencia al tratamiento constituye un problema ampliamente exten- dido en los pacientes con ERC, lo que puede generar graves consecuencias para el paciente como la malnutrición, neuropatías, enfermedad en los huesos, fallo cardíaco e incluso la muerte. Por otra parte la escasa adhesión al régimen terapéutico compromete gravemente su efectividad, disminuye la percepción de bienestar de los pacientes y puede generar costos adicionales al sistema de salud, por ello, las intervenciones deben ir encaminadas a mejorar esta situación y a hacer un importante aporte a nivel de prevención secundaria y terciaria ${ }^{5}$.

La adherencia a la terapia de HD tiene impacto directo en la supervivencia $y$, además, previene descompensaciones agudas entre las sesiones de $\mathrm{HD}^{6}$. El tratamiento integral de estos pacientes requiere el control adecuado en la ingesta de líquidos, dieta y medicación ${ }^{7}$. La ganancia excesiva de peso interdiálisis (inter HD) constituye un factor de riesgo para el incremento de la presión arterial ${ }^{8}$. El comportamiento de no adhesión en el paciente renal se manifiesta de diversas maneras: incumplimiento de la dieta; ingesta de medicamentos y restricción de líquidos y, faltar a las sesiones de HD. Estimar estos criterios de no adhesión implica ciertas dificultades metodológicas, pero, según la bibliografía, los más utilizados son la ganancia de peso inter HD para evaluar el cumplimiento en la restricción de líquidos' el cumplimiento de la dieta y la medicación es estimado por niveles de potasio y fósforo en suero prediálisis (pre $\mathrm{HD}$ ) y el cumplimiento de las sesiones de $\mathrm{HD}^{10}$.

Las investigaciones realizadas han identificado algunas variables que se relacionan con la escasa adherencia: edad avanzada; consumo del tabaco; bajo nivel educativo; depresión y baja percepción del daño; efectos secundarios de los medicamentos; creencias y actitudes del paciente con respecto a la salud; instrucciones poco claras; fallo en la comunicación médico-paciente; complejidad del régimen terapéutico; escasa comprensión de la razón de las terapias y dificultades socioeconómicas ${ }^{11}$.

Uno de los principales problemas que encontramos los profesionales de enfermería que cuidamos del enfermo renal crónico son las dificultades que éstos tienen para cuidarse a sí mismos ${ }^{12}$, favoreciendo así el incumplimiento terapéutico.

\section{Teorías explicativas para mejorar la adherencia de los pacientes a las recomendaciones terapéuticas.}

Teniendo especial atención en la relación del profesional sanitario con el paciente para mejorar la adherencia terapéutica, lleva a reflexionar sobre la importancia de la existencia de un momento y lugar 
determinado en la unidad de HD donde los profesionales de enfermería podamos afianzar esa relación y se facilite la comunicación enfermera-paciente, de forma que podamos tener en cuenta todas las variables que nos van a ayudar a promover la adhesión. Definir y promover la adhesión como la adquisición de hábitos saludables conlleva abordar la adhesión para cada caso concreto desde el análisis de la conducta de cada sujeto en relación a su tratamiento.

El estado actual del conocimiento sobre el comportamiento de adhesión permite comprender la responsabilidad y el papel fundamental que la Enfermería debe de tener en este aspecto del cuidado del enfermo renal. Pero, ¿de qué manera los profesionales de enfermería que trabajamos en una unidad de HD podemos dar una atención integral al paciente y asegurarnos que cada uno de ellos la recibe? El sentimiento, por parte de los profesionales de enfermería que atendemos a este tipo de pacientes día a día, de no abordarlos de forma integral, nos hizo replantearnos la metodología y organización de nuestro quehacer diario y plantear nuevas estrategias.

\section{Objetivo}

Conocer dentro del proceso de personalización de los cuidados, cómo puede influir la asistencia protocolizada del paciente y cuidador principal a la Consulta de Enfermería en la adherencia terapéutica.

\section{Pacientes y Método}

Diseño. Se trata de un estudio observacional longitudinal prospectivo de cohorte.

Muestra. Criterios de inclusión. La muestra del estudio se compone de 42 pacientes en programa de Hemodiálisis en la APES Hospital de Poniente. Se incluyen en el estudio todos aquellos pacientes que permanecen de forma continuada recibiendo tratamiento de HD convencional con bicarbonato en la unidad de HD desde enero 2014 a junio 2014 (6 meses de seguimiento, 42 pacientes).
Procedimiento. Comparación de las variables que miden el cumplimiento terapéutico entre el grupo consulta y el resto de grupos. Intervención $\mathbf{C}$.

Se pone en marcha la consulta de enfermería desde enero de 2014 a junio de 2014. A este periodo de consulta lo denominamos periodo $\mathbf{C}$ y a la consulta en sí la definimos como intervención C. Los pacientes seleccionados para la realización de la consulta son aquellos pertenecientes al Grupo 1 y Grupo 2 por ser la investigadora del estudio la enfermera referente y colaboradora respectivamente de ambos grupos. Al grupo de pacientes que reciben consulta se denomina Grupo A (Grupo 1+2) y el resto de pacientes se engloban en el Grupo B (Grupo 3+4+5+6+7+8). Se recogen los datos de las diferentes variables a estudiar revisando las historias clínicas y mediante la entrevista con el paciente y/o el cuidador principal. (Figura 1).

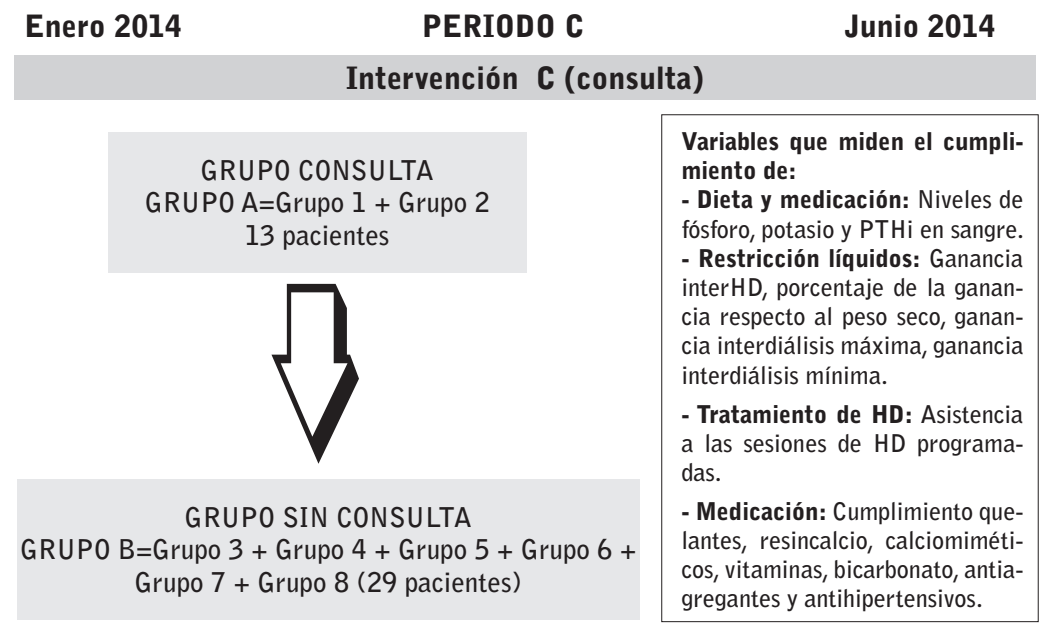

Figura 1. Comparación de las variables que miden el cumplimiento terapéutico entre el grupo consulta y el resto de grupos. Intervención C.
Variables que miden el cumpli-
miento de:
- Dieta y medicación: Niveles de
fósforo, potasio y PTHi en sangre.
- Restricción líquidos: Ganancia
interHD, porcentaje de la ganan-
cia respecto al peso seco, ganan-
cia interdiálisis máxima, ganancia
interdiálisis mínima.
- Tratamiento de HD: Asistencia
a las sesiones de HD programa-
das.
- Medicación: Cumplimiento que-
lantes, resincalcio, calciomiméti-
cos, vitaminas, bicarbonato, antia-
gregantes y antihipertensivos.

\section{Intervención C. Programación de la Consulta de En- fermería.}

Creamos la consulta de enfermería de HD utilizando para esto una sala que se había habilitado en la unidad de HD, para citar y entrevistar a los pacientes asignados.

Estructura consulta: Ambas enfermeras referentes (Grupo 1 y 2) citan a los 13 pacientes una vez tengan los resultados de las analíticas mensuales y la hoja de tratamiento médico. A cada paciente se le cita a una hora para que acuda con su cuidador principal y se le pide que traiga las cajas con la medicación prescrita. La duración aproximada de la consulta es de 1 hora y está estructurada de la forma que se muestra en la 
tabla 1. Todos los pacientes firman el consentimiento informado para utilizar sus datos con fines científicos.

Tabla 1. Estructura de la consulta de Enfermería.

ESTRUCTURA CONSULTA
1. Presentación.
2. Objetivo de la consulta. Explicamos la finalidad y se pide el
consentimiento para utilizar sus datos en el estudio.
3. Revisión del tratamiento farmacológico. Explicamos las
dudas existentes, forma de administración, olvidos, efectos
secundarios.... Valoramos si es cumplidor del tratamiento y
se registra en la hoja habilitada para esto.
4. Realización de un test de hábitos y estilos de vida en el
paciente renal diseñado para detectar el déficit de cono-
cimientos en diferentes aspectos. Dependiendo de los re-
sultados del test y de la analítica mensual, realizaremos la
educación sanitaria sobre todo orientada a la dieta, ase-
gurando que sea lo más individualizada posible y evitando
prohibiciones innecesarias.
5. Ofrecer la información necesaria acompañada de soporte
escrito (trípticos, folletos, guías de recomendaciones, etc.).
6res que puedan llegar a tener como consecuencia del pro-
ceso de la enfermedad.
9. Fomentuación de los objetivos del plan de cuidados.
7. Resolver aquellas dudas que tenga tanto el cuidador princi-
pal como el paciente.

10. Pactamos objetivos y metas con cada uno de los pacientes, dependiendo del aspecto que tenga que mejorar cada uno; esto se evaluará el siguiente mes.

\section{Definición de las variables del estudio}

El criterio de adherencia terapéutica es evaluado a través de varios indicadores bioquímicos y clínicos, los cuales fueron recogidos de las historias clínicas de cada paciente registrándose en una hoja diseñada para tal fin. Teniendo en cuenta el criterio médico por parte del Coordinador de Nefrología, y basándonos en las Guías SEN de Centros de Hemodiálisis ${ }^{13}$ y el Proceso Asistencial Integrado ${ }^{14}$, se definieron los criterios de adhesión en general del paciente y sobre cada uno de los indicadores incluidos en el estudio. Los indicadores clínicos y bioquímicos se registraron fueron los siguientes: ganancia interdiálisis (GI); porcentaje de la ganancia sobre su peso seco (\% PS); tensión arterial pre hemo- diálisis (TA preHD) y tensión arterial post hemodiálisis (TA post HD); niveles de potasio (K); calcio ( $\mathrm{Ca}$ ); sodio $(\mathrm{Na})$; fósforo $(\mathrm{P})$; hemoglobina $(\mathrm{Hb})$ y hormona paratiroidea (PTHi), así como la asistencia cumplida y completa a las sesiones de hemodiálisis. Se recogieron diferentes variables para medir el cumplimiento del tratamiento farmacológico por tipo de medicamentos.

\section{Análisis de los datos.}

EI análisis de los datos se realiza mediante el paquete estadístico SPSS v16. Los resultados se expresan para las variables cuantitativas en forma de media y desviación típica y en forma de frecuencias y porcentajes para las variables cualitativas. Se considera significativo $p$ $\leq 0.05$.

\section{Resultados}

Se estudiaron un total de 42 pacientes (64\% hombres) con una edad media de $56.7 \pm 17.7$ años y un rango de edad entre 88 y 24 años. Todos los pacientes reciben HD convencional con bicarbonato y dializadores de alta permeabilidad.

Los resultados del análisis de las variables cuantitativas que miden el cumplimiento terapéutico entre grupos, se exponen tras la comparación de dos muestras independientes (t de Student).

Analizamos los niveles de fósforo, potasio y PTHi en ambos grupos, encontrando diferencias significativas en los niveles de P y PTHi. Figura 2. Tabla 2.

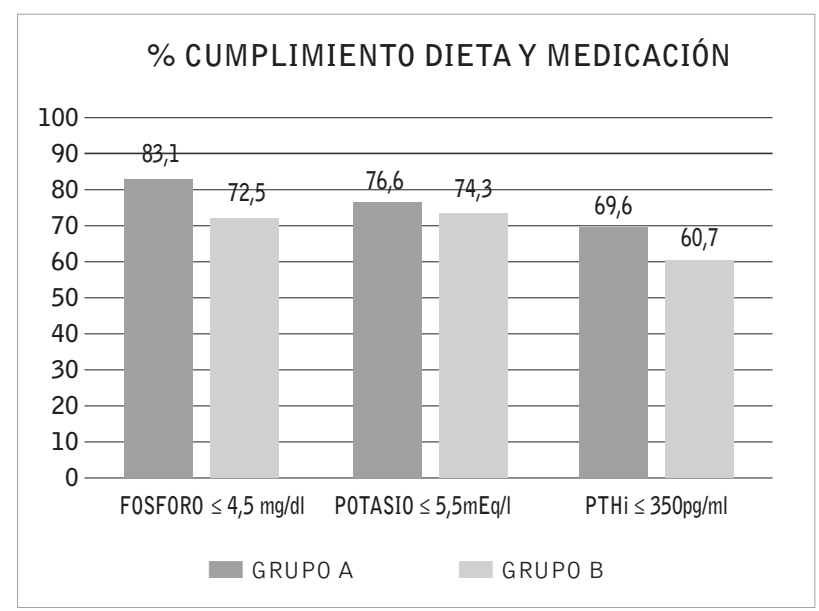

Figura 2. Porcentaje de cumplimiento de dieta y mediación basado en los niveles de fósforo, potasio y PTHi. 
Tabla 2. Comparación de medias para los niveles de P y PTHi entre ambos grupos.

\begin{tabular}{|l|c|c|}
\hline & $\begin{array}{c}\text { Niveles de Fósforo } \\
\text { Grupo A }\end{array}$ & $\begin{array}{c}\text { Niveles de Fósforo } \\
\text { Grupo B }\end{array}$ \\
\hline Media & $3.94 \mathrm{mg} / \mathrm{dl}$ & $4.30 \mathrm{mg} / \mathrm{dl}$ \\
\hline Desviación estándar & 1.08 & 1.44 \\
\hline $\mathrm{n}^{\circ}$ pacientes & 13 & 29 \\
\hline $\mathrm{N}$ & 77 & 171 \\
\hline & Niveles de PTHi & Niveles de PTHi \\
Grupo A & Grupo B \\
\hline Media & $282.43 \mathrm{pg} / \mathrm{ml}$ & $375.05 \mathrm{pg} / \mathrm{ml}$ \\
\hline Desviación estándar & 182.26 & 343.95 \\
\hline $\mathrm{n}^{\circ}$ pacientes & 13 & 29 \\
\hline $\mathrm{N}$ & 46 & 112 \\
\hline
\end{tabular}

Al analizar la ganancia interdialisis y el \% Peso Seco, no encontramos diferencias significativas.

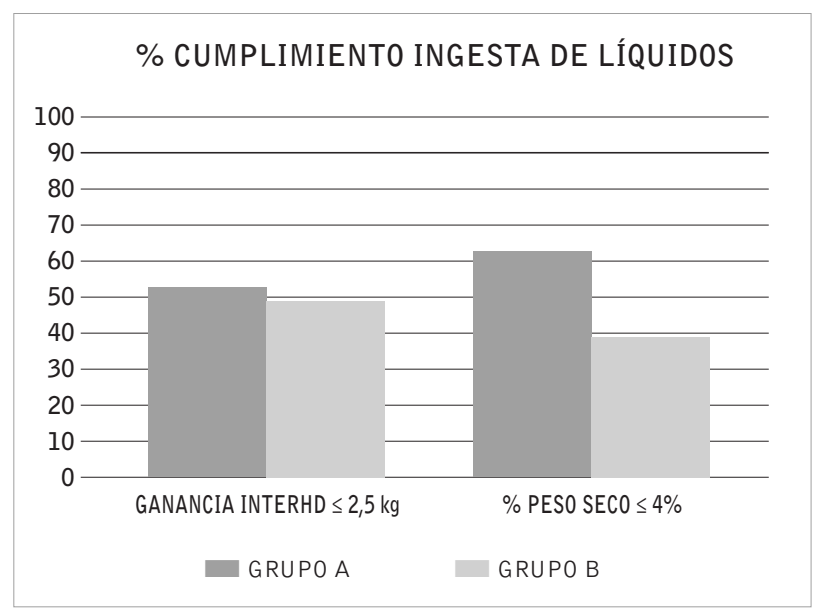

Figura 3. Porcentaje de cumplimiento de la ingesta de líquidos basado en la ganancia interdialisis y el \%PS.
Los resultados del análisis de las variables cualitativas que miden el cumplimiento terapéutico entre grupos, se exponen tras la comparación de dos muestras independientes ( $\mathrm{Ji}$ cuadrado).

Analizamos el cumplimiento farmacológico por tipo de medicamentos entre ambos grupos encontrando diferencias significativas para los quelantes del fósforo, resincalcio, calcimiméticos, bicar$p=0,030$ bonato y vitaminas. Figura 4

\section{Discusión}

Tras el análisis de las diferentes variables cuantitativas y cualitativas seleccionadas para evaluar la adherencia terapéutica en los pacientes que asisten a la consulta de enfermería, Intervención C, podemos observar que se produce una mejora en la adherencia terapéutica ya que al comparar las variables entre los diferentes grupos se encuentran diferencias estadísticamente significativas para el fósforo y la PTHi, pero no en los niveles de potasio en sangre, ganancia interdiálisis y $\%$ PS.

Al comparar los niveles de fósforo entre el Grupo A y Grupo B se encuentran diferencias estadísticamente significativas. Esto es debido a que la media de fósforo en el Grupo A es de $3.94 \mathrm{mg} / \mathrm{dl}$ con un porcentaje de paciente cumplidores de un $83.1 \%$, frente a una media de fósforo de $4.3 \mathrm{mg} / \mathrm{dl}$ en el Grupo B con un porcentaje de cumplidores de un $72.5 \%$.

Al analizar las cifras de potasio no ocurre lo mismo, incluso en el Grupo A la media es ligeramente más elevada, pudiendo estar relacionado con la individualización de la dieta, al fomentar el tratamiento adecuado de los alimentos ricos en potasio en vez de prohibirlos radicalmente, pero nos encontramos dentro del rango normal.

Respecto a los niveles de PTHi, si existen diferencias significativas entre los dos grupos, teniendo una media de $282.43 \mathrm{pg} / \mathrm{ml}$ en el Grupo A frente a un $375.05 \mathrm{pg} / \mathrm{ml}$ en el Grupo $B$, estando relacionado directamente con la toma de calciomiméticos.

Figura 4. Porcentaje de cumplimiento farmacológico en ambos grupos. 
No se encuentran diferencias significativas al comparar las variables que miden la restricción de líquidos, es decir la ganancia interdiálisis y el \%PS.

Al analizar las variables cualitativas referentes al cumplimiento del tratamiento farmacológico entre los dos grupos podemos observar que si existen diferencias significativas para los quelantes del fósforo, el poliestireno, los calciomiméticos, las vitaminas y el bicarbonato, medicamentos específicos del tratamiento farmacológico de este tipo de pacientes. No se encuentran diferencias significativas para los antihipertensivos y los antiagregantes plaquetarios, ya que todos los pacientes son muy buenos cumplidores para este tipo de medicamentos, coincidiendo con otros estudios ${ }^{16}$.

Con estos resultados podemos decir que la consulta de enfermería hace que mejore la adherencia terapéutica en cuanto a la dieta y la medicación. Esto puede estar relacionado con un mayor tiempo de dedicación al tratamiento del paciente, y mayor contacto con el cuidador principal. Este contexto favorece aquellas intervenciones que dependen en gran medida de una adecuada educación sanitaria donde el contexto tiene gran importancia ${ }^{17}$.

Habitualmente, cuando entrevistamos a un paciente en la sala de HD o se realizamos educación sanitaria se producen muchas interferencias en la comunicación ya que a la misma vez atendemos a otros pacientes y se presentan frecuentemente problemas que tenemos que resolver de forma inmediata, por tanto, ni entrevistamos al paciente ni realizamos educación sanitaria de forma correcta. Con la consulta de enfermería se pretende evitar esto, y darle el valor y el tiempo que tienen todas estas intervenciones enfermeras que son parte del tratamiento de la enfermedad. Diversos estudios sobre la adherencia terapéutica demuestran que la especificación periódica por escrito de los fármacos que tienen que recibir y las dosis mejora el cumplimiento del tratamiento farmacológico ${ }^{18-20}$.

El personal de enfermería debe asegurarse siempre que el paciente reciba las instrucciones terapéuticas correctamente escritas, revisarlas y verificarlas con él para poder hacer un ajuste de los niveles y comprensión, aunque la enseñanza del paciente debe de ir más allá de una información correcta. Un reto mayor es el de motivarlo y corresponsabilizarlo para lograr la adquisición de conductas implicadas en sus autocuidados ${ }^{18}$.

Es muy importante hacer un seguimiento constante de la toma de la medicación y poder evitar el mayor número posible de errores. Los programas educativos en este sentido pueden beneficiar a todos aquellos pacientes susceptibles de este tipo de intervención que debe efectuarse de forma continua y si grandes intervalos de tiempo.

Está demostrado que el cumplimiento terapéutico disminuye las estancias hospitalarias, reduciendo por tanto las complicaciones y facilitando una mejor evolución de la enfermedad ${ }^{12}$. El papel de la enfermería es fundamental a la hora del cumplimiento del régimen terapéutico.

\section{Conclusiones}

La consulta de enfermería para el paciente renal en programa de HD mejora la adherencia terapéutica en cuanto a la dieta y la medicación.

\author{
Recibido: 13 noviembre 2016 \\ Revisado: 5 diciembre 2016 \\ Modificado: 28 abril 2017 \\ Aceptado: 15 mayo 2017
}

\section{Bibliografía}

1. González V, Lobo N. Calidad de vida en los pacientes con insuficiencia renal crónica terminal en tratamiento sustitutivo de hemodiálisis. Aproximación a un proyecto integral de apoyo. Revista de la Sociedad Española de Enfermería Nefrológica 2001; 4:6-12.

2. García F, Fajardo C, Guevara R, Gonzáles V, Hurtado A. Mala adherencia a la dieta en hemodiálisis: papel de los síntomas ansiosos y depresivos. Nefrología 2002; 22:245-252.

3. García AM, Leiva F, Martos F, García AJ, Prados D, Sánchez F. ¿Cómo diagnosticar el cumplimiento terapéutico en Atención Primaria? Medicina de Familia 2000; 1:13-19.

4. Cvengros BS, Christensen PD, Lawton MD.The role of perceived control and preference for control in adherence to a chronic medical regimen. Ann Behav Med 2004; 27(3):155-161.

5. Organización Mundial de la Salud. Adherencia a los tratamientos a largo plazo: Pruebas para la acción. Washington, DC; 2004. 
6. Morduchowicz G. Compliance in hemodiálisis patients: a multivariate regression analysis. Nephron 1993; 64 (3):365-8.

7. Leggat JE, Orzol SM, Hulbert-Shearon TE. Noncompliance in hemodialysis. Predictors and survival analysis. Am J Kidney Dis 1998; 32:139145.

8. Rahman M, Ping Fu Fu, Ashwini R. Interdialytic weight gain, compliance with dialysis regimen an age are independent predictors of blood pressure in hemodialysis patient. Am J Kidney Dis 2000; 35:257-265.

9. Khechane N, Mwaba K. Treatment adherence and coping with stress among Black South African Haemodialysis patients. Social Behaviour and Personality Journal 2004; 32(8):777-782.

10. Hailey B, Moss S. Compliance behaviour in patients undergoing haemodialysis: A review of the literature. Psychology Health and medicine 2000; 5:395-406.

11. Kaveh K, Kimmel P. Compliance in hemodialysis patients: multidimensional measures in search of a gold standard. American Journal of Kidney Diseases 2001; 37(2):244-266.

12. Vila M, García N, Gómez AM, García R, Tejuca $M$, Tejuca A. Cumplimentación terapéutica de pacientes en diálisis. Libro de Comunicaciones presentadas en el XXXII Congreso Nacional de la Sociedad Española de Enfermería Nefrológica. Cádiz 2007: 173-177.

13. Guías SEN. Guías de centros de hemodiálisis. Revista de la Sociedad Española de Nefrología. 2006; suplemento 8 vol. 26.
14. Consejería de Salud. Tratamiento sustitutivo de la insuficiencia renal crónica: diálisis y trasplante renal: Proceso Asistencial Integrado. Sevilla: Consejería de Salud; 2005.

15. Sezer S, Ozdemir FN, Arat Z, Perim 0, Turan $M$, Haberal $M$. The association of interdialytic weight gain with nutricional parameters and mortality risk in hemodialysis patients. Ren Fail. 2002; 24:37-48

16. Galera A, Casado S. Programa educacional para un mejor control dietético y farmacológico de los pacientes en hemodiálisis. Libro de Comunicaciones presentadas al XIII Congreso Nacional de la Sociedad Española de Enfermería Nefrológica, 1988:108-113.

17. López-Gómez JM, Villaverde M, Jofré R, RodríguezBenítez P, Pérez García R. Interdialytic weight gain as a marker of blood pressure, nutrition, and survival in hemodialysis patients. Kidney Int. 2005; (suppl.93):S63-68.

18. Sezer S, Ozdemir FN, Arat Z, Perim 0, Turan $M$, Haberal $M$. The association of interdialytic weight gain with nutricional parameters and mortality risk in hemodialysis patients. Ren Fail. 2002; 24:37-48

19. Galera A, Casado S. Programa educacional para un mejor control dietético y farmacológico de los pacientes en hemodiálisis. Libro de Comunicaciones presentadas al XIII Congreso Nacional de la Sociedad Española de Enfermería Nefrológica, 1988:108-113.

20. Tazon A, Aseguinolaza, L y García-Campayo. J. Ciencias psicosociales. Barcelona: Masson; 1997.p.152-159. 